\title{
Torricellian points in normed linear spaces
}

\author{
Sever S Dragomir ${ }^{1,2}$, Dan Comănescu ${ }^{3}$ and Eder Kikianty ${ }^{2 *}$
}

\author{
${ }^{*}$ Correspondence: \\ eder.kikianty@wits.ac.za \\ ${ }^{2}$ School of Computational and \\ Applied Mathematics, University of \\ the Witwatersrand, Private Bag 3, \\ Wits, Johannesburg, 2050, South \\ Africa \\ Full list of author information is \\ available at the end of the article
}

\begin{abstract}
Given a set of $n$ (distinct) points $\mathcal{A}$ in a normed space, we consider the set of Torricellian points, that is, the set of points which minimises the sum of distances to the points in $\mathcal{A}$. We introduce the Torricellian functional associated to a set of distinct points $\mathcal{A}$, which calculates the sum of distances of a point $x$ to the points in $\mathcal{A}$. The Torricellian point is defined as the infimum (over all vectors) of this functional. We discuss the existence of Torricellian points in reflexive normed spaces, non-expansive subspaces and evidently, inner product spaces. A case for collinear points is given and is utilised to characterise strict convexity. For a non-collinear case, it is shown that the set of Torricellian points contains a unique point when the space is strictly convex. However, we show that the uniqueness of Torricellian point of a non-collinear set does not characterise strict convexity. We consider a particular example of the Torricellian problem in a space endowed with the Taxicab geometry.

MSC: 46B20; 49J27
\end{abstract}

Keywords: Fermat point; Torricellian point; characterisation of strictly convex spaces; Taxicab geometry

\section{Introduction}

In 1643, Fermat raised the following problem [1]:

'Given three distinct points in the plane, find the (unique) point having the minimal sum of distances to these three points.'

This problem was first solved by Torricelli, whose result was published by his pupil Viviani in 1659. This (unique) point is often referred to as the Fermat point, Fermat-Torricellian point, or Torricellian point. In this text, we refer to it as the Torricellian point. The solution is as follows: if all angles of the triangle are less than $\frac{2 \pi}{3}$, then the Torricellian point is the interior point from which each side subtends an angle of $\frac{2 \pi}{3}$. If one of the angles is greater than $\frac{2 \pi}{3}$, then the Torricellian point lies at the obtuse angled vertex.

A straightforward generalisation of this problem is as follows: given $n$ points $a_{1}, \ldots, a_{n} \in$ $\mathbb{R}^{d}(d \geq 1)$, find $x_{0} \in \mathbb{R}^{d}$ which minimises the sum of distances to all the $n$ points. It is a well-known result that when $d=1$ (case of collinear points), the Torricellian point $x_{0}$ coincides with the centre point of the set $\left\{a_{1}, \ldots, a_{n}\right\}$ when $n$ is odd, i.e. $x_{0}=a_{k}$, where $k=\frac{(n+1)}{2}$; and $x_{0}$ is any point lying between $a_{k}$ and $a_{k+1}$, where $k=\frac{n}{2}$ when $n$ is even (cf. Dalla [1] and Simons [2]). Given the large number of literature, we refer the reader to the survey paper by Kupitz and Martini [3] and Section II.8 of the book by Boltyanski et al. [4] for further reading on this topic.

Torricelli's problem can be defined in any metric spaces, but if we wanted to have nice properties in characterising Torricellian points, we would have to restrict ourselves to the

\section{Springer}

(c) 2013 Dragomir et al.; licensee Springer. This is an Open Access article distributed under the terms of the Creative Commons Attribution License (http://creativecommons.org/licenses/by/2.0), which permits unrestricted use, distribution, and reproduction in any medium, provided the original work is properly cited. 
case of normed linear spaces. The set of Torricellian points can be utilised to characterise strictly convex spaces and inner product spaces. In Martini et al. [5], a generalisation in Minkowski spaces is considered. It is shown that the Torricellian point is unique if and only if the Minkowski space is strictly convex. Dragomir et al. in [6] considered and solved Torricelli's problem in a real inner product space of dimension greater than 1 . A generalisation to $n$ arbitrary points in a real inner product space is considered in Dragomir and Comănescu [7].

The aim of this paper is to consider the generalisation of the Torricellian points in the settings of normed spaces ( $c f$. Section 2). We introduce the Torricellian functional associated to a set of distinct points $\mathcal{A}$, which calculates the sum of distances of a point $x$ to the points in $\mathcal{A}$. The Torricellian point is defined as the infimum (over all vectors) of this functional. We discuss the existence of the Torricellian points in Section 3. We recall some known results from the papers by Veselý [8], in which the author discussed the Torricellian set in reflexive normed spaces; and also Papini and Puerto [9] for some results in Banach spaces. We also consider the existence of Torricellian point for a set of distinct points which spans a non-expansive subspace; and as a corollary, we consider the existence in inner product spaces which recaptures the results of Dragomir and Comănescu [7]. In Section 4, we consider the case of collinear points in normed spaces; and in particular, strictly convex spaces and inner product spaces. In Section 5 we show that for the case of non-collinear points, the Torricellian point is unique in strictly convex spaces and inner product spaces. However, the uniqueness of Torricellian points does not characterise strict convexity. Finally, in Section 6, we consider an example of the Torricellian problem in spaces endowed with the Taxicab geometry.

\section{Definitions and notation}

This section serves as a reference point for definitions and notation that are used in the paper.

Definition 1.1 Let $X$ be a (real) vector space, and let $T$ be a functional on $X$. The left-sided and right-sided Gâteaux derivatives of a functional $T$ at $x_{0}$ on direction $x$ are defined as follows:

$$
\left(V_{-} T\right)\left(x_{0}\right) \cdot x:=\lim _{t \rightarrow 0-} \frac{T\left(x_{0}+t x\right)-T\left(x_{0}\right)}{t}
$$

and

$$
\left(V_{+} T\right)\left(x_{0}\right) \cdot x:=\lim _{t \rightarrow 0+} \frac{T\left(x_{0}+t x\right)-T\left(x_{0}\right)}{t} .
$$

When $V_{-} T=V_{+} T$, we say that $T$ is Gâteaux differentiable and denote the derivative as $V T$.

Definition 1.2 Let $(X,\|\cdot\|)$ be a (real) normed space. The (real-valued) mappings $\langle\cdot, \cdot\rangle_{i}$ and $\langle\cdot, \cdot\rangle_{s}$ defined on $X \times X$ given by:

$$
\langle x, y\rangle_{i}=\lim _{t \rightarrow 0-} \frac{\|y+t x\|^{2}-\|y\|^{2}}{2 t}, \quad\langle x, y\rangle_{s}=\lim _{t \rightarrow 0+} \frac{\|y+t x\|^{2}-\|y\|^{2}}{2 t}
$$

are called the inferior and superior semi-inner products. 
For more details connected with these norm derivatives, we refer to Dragomir [10] where further references are given. For the sake of completeness, we list some usual properties of these semi-inner products:

(i) $\langle x, x\rangle_{p}=\|x\|^{2}$ for all $x \in X$;

(ii) $\langle-x, y\rangle_{s}=\langle x,-y\rangle_{s}=-\langle x, y\rangle_{i}$ if $x, y \in X$;

(iii) $\langle\alpha x, \beta y\rangle_{p}=\alpha \beta\langle x, y\rangle_{p}$ for all $x, y \in X$ and $\alpha, \beta \geq 0$;

(iv) $\langle\alpha x+y, x\rangle_{p}=\alpha\langle x, x\rangle_{p}+\langle y, x\rangle_{p}$ if $x, y \in X, \alpha \in \mathbb{R}$;

(v) $\langle x+y, z\rangle_{p} \leq\|x\|\|z\|+\langle y, z\rangle_{p}$ for all $x, y, z \in X$;

(vi) the space $(X,\|\cdot\|)$ is smooth, i.e. the norm $\|\cdot\|$ is Gâteaux differentiable on $X \backslash\{0\}$ iff $\langle x, y\rangle_{i}=\langle x, y\rangle_{s}$ for all $x, y \in X$; or iff $\langle\cdot, \cdot\rangle_{p}$ is linear in the first variable;

where $p=s$ or $p=i$.

\section{Generalisation of Torricellian points}

Let $(X,\|\cdot\|)$ be a real normed linear space, let $n \geq 1$ be a natural number and let $\mathcal{A}=$ $\left\{a_{1}, \ldots, a_{n}\right\}$ be a set of $n$ distinct points in $X$. Throughout the paper, we denote $\overline{1, n}=1, \ldots, n$. The functional

$$
T=T_{\mathcal{A}}: X \rightarrow[0, \infty), \quad T(x):=\sum_{i=1}^{n}\left\|x-a_{i}\right\|
$$

will be called the Torricellian functional associated with $\mathcal{A}$. The main properties of this mapping can be summarised in the following proposition. We refer to Dragomir and Comănescu [7, Proposition 1$]$ for the proof.

Proposition 2.1 With the above assumptions, we have:

(i) $T$ is nonlinear on $X$;

(ii) $T$ is continuous on $X$ in the norm topology;

(iii) $T$ is nonnegative and $\lim _{\|x\| \rightarrow \infty} T(x)=\infty$;

(iv) $T$ is convex on $X$.

Concerning the Gâteaux derivative of $T$, we have the following results.

Proposition 2.2 With the above assumption, we have

$$
\left(V_{ \pm} T\right)\left(x_{0}\right) \cdot x= \begin{cases}\sum_{i=1}^{n} \frac{\left\langle x, x_{0}-a_{i}\right\rangle_{s(i)}}{\left\|x_{0}-a_{i}\right\|} & \text { if } x_{0} \notin \mathcal{A} ; \\ \sum_{i=1}^{n} \frac{\left\langle x, a_{j}-a_{i}\right\rangle_{s}(i)}{\left\|a_{j}-a_{i}\right\|} \pm\|x\| & \text { if } x_{0}=a_{j} \text { for } j \in\{1, \ldots, n\}\end{cases}
$$

for all $x \in X$.

Proof Suppose that $x_{0} \notin \mathcal{A}$. Then we have

$$
\begin{aligned}
\left(V_{ \pm} T\right)\left(x_{0}\right) \cdot x & :=\sum_{i=1}^{n} \lim _{t \rightarrow 0 \pm} \frac{\left\|x_{0}-a_{i}+t x\right\|-\left\|x_{0}-a_{i}\right\|}{t} \\
& =\sum_{i=1}^{n} \lim _{t \rightarrow 0 \pm}\left[\frac{\left\|x_{0}-a_{i}+t x\right\|^{2}-\left\|x_{0}-a_{i}\right\|^{2}}{2 t} \cdot \frac{2}{\left\|x_{0}-a_{i}+t x\right\|+\left\|x_{0}-a_{i}\right\|}\right] \\
& =\sum_{i=1}^{n} \frac{\left\langle x, x_{0}-a_{i}\right\rangle_{s(i)}}{\left\|x_{0}-a_{i}\right\|}
\end{aligned}
$$


for all $x \in X$. Let $x_{0}=a_{j}$ with a fixed $j \in\{1, \ldots, n\}$. Then

$$
\begin{aligned}
\left(V_{ \pm} T\right)\left(x_{0}\right) \cdot x & :=\sum_{\substack{i=1 \\
i \neq j}}^{n} \lim _{t \rightarrow 0 \pm} \frac{\left\|a_{j}-a_{i}+t x\right\|-\left\|a_{j}-a_{i}\right\|}{t}+\lim _{t \rightarrow 0 \pm} \frac{|t|\|x\|}{t} \\
& =\sum_{\substack{i=1 \\
i \neq j}}^{n} \frac{\left\langle x, a_{j}-a_{i}\right\rangle_{s(i)}}{\left\|a_{j}-a_{i}\right\|} \pm\|x\|
\end{aligned}
$$

and this completes the proof.

Corollary 2.3 Let $(X,\|\cdot\|)$ be a smooth normed space and denote by $[\cdot, \cdot]:=\langle\cdot, \cdot\rangle_{s}=\langle\cdot, \cdot\rangle_{i}$ the semi-inner product which generates the norm $\|\cdot\|$. Then $T$ is Gâteaux differentiable on $X \backslash \mathcal{A}$ and

$$
(V T)\left(x_{0}\right) \cdot x=\sum_{i=1}^{n} \frac{\left[x, x_{0}-a_{i}\right]}{\left\|x_{0}-a_{i}\right\|} \quad \text { if } x_{0} \notin \mathcal{A}
$$

for all $x \in X$.

Remark 2.4 Corollary 2.3 also holds for any inner product space $(X,\langle\cdot, \cdot\rangle)$ as it is a smooth space. This recaptures the results in Dragomir and Comănescu [7, Proposition 3].

Concerning the strict convexity property of $T$, we have the following proposition. We refer to [7, Proposition 2] for the proof.

Proposition 2.5 Let $(X,\|\cdot\|)$ be a strictly convex normed linear space. If $\mathcal{A}=\left\{a_{1}, \ldots, a_{n}\right\}$ with $n \geq 3$ is a set of non-collinear points in $X$, then $T$ is strictly convex on $X$.

Now, we formally define the Torricellian points for a given set of points.

Definition 2.6 Let $(X,\|\cdot\|)$ be a real normed linear space, let $n \geq 1$ be a natural number and let $\left\{a_{1}, \ldots, a_{n}\right\}$ be a set of distinct elements in $X$. The point $x_{0} \in X$ will be called a Torricellian point of the set $\left\{a_{1}, \ldots, a_{n}\right\}$ if it minimises the Torricellian functional $T$, i.e.

$$
T\left(x_{0}\right)=\inf _{x \in X} T(x)
$$

or, equivalently,

$$
\sum_{i=1}^{n}\left\|x_{0}-a_{i}\right\| \leq \sum_{i=1}^{n}\left\|x-a_{i}\right\| \quad \text { for all } x \in X
$$

The set of all Torricellian points of $\left\{a_{1}, \ldots, a_{n}\right\}$ will be denoted by $\mathcal{T}_{X}\left\{a_{1}, \ldots, a_{n}\right\}$.

We describe the main properties of $\mathcal{T}_{X}\left\{a_{1}, \ldots, a_{n}\right\}$ for any $\left\{a_{1}, \ldots, a_{n}\right\} \subset X$ in the next proposition.

Proposition 2.7 For any $\left\{a_{1}, \ldots, a_{n}\right\} \subset X$, we have that $\mathcal{T}_{X}\left\{a_{1}, \ldots, a_{n}\right\}$ is a convex, closed and bounded subset of the normed linear space $X$. 
Proof Assume that $\mathcal{T}_{X}\left\{a_{1}, \ldots, a_{n}\right\}$ is nonempty, as the proof is vacuous when the set is empty. If $x_{1}, x_{2} \in \mathcal{T}_{X}\left\{a_{1}, \ldots, a_{n}\right\}$ and $\lambda \in[0,1]$, then

$$
T\left(\lambda x_{1}+(1-\lambda) x_{2}\right) \leq \lambda T\left(x_{1}\right)+(1-\lambda) T\left(x_{2}\right) \leq \lambda T(x)+(1-\lambda) T(x)=T(x)
$$

for all $x \in X$, which shows that $\left[x_{1}, x_{2}\right] \subset \mathcal{T}_{X}\left\{a_{1}, \ldots, a_{n}\right\}$, i.e. the set $\mathcal{T}_{X}\left\{a_{1}, \ldots, a_{n}\right\}$ is convex.

Now, let $x_{0} \in \mathcal{T}_{X}\left\{a_{1}, \ldots, a_{n}\right\}$ and put $\alpha_{0}=T\left(x_{0}\right)$. Then $\mathcal{T}_{X}\left\{a_{1}, \ldots, a_{n}\right\}=T^{-1}\left(\left\{\alpha_{0}\right\}\right)$ which is a closed set as the mapping is continuous in the norm topology of $X$ and $\left\{\alpha_{0}\right\}$ is closed in $\mathbb{R}$.

Let $x_{0} \in \mathcal{T}_{X}\left\{a_{1}, \ldots, a_{n}\right\}$ and put $\alpha=T\left(x_{0}\right)=\inf _{x \in X} T(x)$. Then

$$
\alpha=T\left(x_{0}\right)=\sum_{i=1}^{n}\left\|x_{0}-a_{i}\right\| \geq \sum_{i=1}^{n}\left|\left\|x_{0}\right\|-\left\|a_{i}\right\|\right| \geq n\left\|x_{0}\right\|-\sum_{i=1}^{n}\left\|a_{i}\right\|,
$$

which gives us

$$
\left\|x_{0}\right\| \leq \frac{\alpha+\sum_{i=1}^{n}\left\|a_{i}\right\|}{n}
$$

and thus proves the boundedness of the set $\mathcal{T}_{X}\left\{a_{1}, \ldots, a_{n}\right\}$.

We introduce the following definition.

Definition 2.8 The Torricellian point $x_{0} \in \mathcal{T}_{X}\left\{a_{1}, \ldots, a_{n}\right\}$ is called segmentally inferior relating to the set $\mathcal{T}_{X}\left\{a_{1}, \ldots, a_{n}\right\}$ if there exist $x_{1}, x_{2} \in \mathcal{T}_{X}\left\{a_{1}, \ldots, a_{n}\right\}, x_{1} \neq x_{2}$, such that $x_{0} \in$ $\left(x_{1}, x_{2}\right)$.

Proposition 2.9 If the point $x_{0} \in \mathcal{T}_{X}\left\{a_{1}, \ldots, a_{n}\right\}$ is segmentally inferior to $\mathcal{T}_{X}\left\{a_{1}, \ldots, a_{n}\right\}$, then there exist $x_{1}, x_{2} \in \mathcal{T}_{X}\left\{a_{1}, \ldots, a_{n}\right\}$ such that $T$ is Gâteaux differentiable on $x_{0}$ in the $\left(x_{2}-x_{1}\right)$ direction and

$$
(V T)\left(x_{0}\right) \cdot\left(x_{2}-x_{1}\right)=0 .
$$

Proof Let $x_{0}$ be a segmentally inferior point of $\mathcal{T}_{X}\left\{a_{1}, \ldots, a_{n}\right\}$. Then there exist $x_{1}, x_{2}, x_{1} \neq$ $x_{2}$, so that $x_{0} \in\left(x_{1}, x_{2}\right)$, i.e. $x_{0}=s x_{1}+(1-s) x_{2}$ with $s \in(0,1)$. If $x_{0}=s x_{1}+(1-s) x_{2}$ with $s \in(0,1)$, then $x_{0}+t\left(x_{2}-x_{1}\right) \in\left[x_{1}, x_{2}\right]$ if and only if $t \in(1,1-s)$. For $t \in(1-s, s)$ we have $T\left(x_{0}+t\left(x_{2}-x_{1}\right)\right)=T\left(x_{0}\right)$ and hence

$$
(V T)\left(x_{0}\right) \cdot\left(x_{2}-x_{1}\right)=\lim _{t \rightarrow 0} \frac{T\left(x_{0}+t\left(x_{2}-x_{1}\right)\right)-T\left(x_{0}\right)}{t}=0,
$$

which completes the proof.

Corollary 2.10 The points $a_{j}$ with $j=\overline{1, n}$ cannot be segmentally inferior to the set $\mathcal{T}_{X}\left\{a_{1}, \ldots, a_{n}\right\}$.

Proof Let us assume that $a_{j}$ is segmentally inferior to $\mathcal{T}_{X}\left\{a_{1}, \ldots, a_{n}\right\}, j \in\{1, \ldots, n\}$. Then there exist $x_{1}, x_{2} \in \mathcal{T}_{X}\left\{a_{1}, \ldots, a_{n}\right\}$ such that $a_{j} \neq x_{1}, x_{2}$ and $a_{j} \in\left(x_{1}, x_{2}\right)$. By Proposition 2.9 
we have

$$
\left(V_{+} T\right)\left(a_{j}\right)\left(x_{2}-x_{1}\right)=\left(V_{-} T\right)\left(a_{j}\right)\left(x_{2}-x_{1}\right)=0 .
$$

By Proposition 2.2 we have

$$
0=\left(V_{+} T\right)\left(a_{j}\right)\left(x_{2}-x_{1}\right)=\sum_{\substack{i=1 \\ i \neq j}}^{n} \frac{\left\langle x_{2}-x_{1}, a_{j}-a_{i}\right\rangle_{s}}{\left\|a_{j}-a_{i}\right\|}+\left\|x_{2}-x_{1}\right\|
$$

and

$$
0=\left(V_{-} T\right)\left(a_{j}\right)\left(x_{2}-x_{1}\right)=\sum_{\substack{i=1 \\ i \neq j}}^{n} \frac{\left\langle x_{2}-x_{1}, a_{j}-a_{i}\right\rangle_{i}}{\left\|a_{j}-a_{i}\right\|}-\left\|x_{2}-x_{1}\right\|,
$$

from which we get

$$
\sum_{\substack{i=1 \\ i \neq j}}^{n} \frac{\left\langle x_{2}-x_{1}, a_{j}-a_{i}\right\rangle_{s}}{\left\|a_{j}-a_{i}\right\|}-\sum_{\substack{i=1 \\ i \neq j}}^{n} \frac{\left\langle x_{2}-x_{1}, a_{j}-a_{i}\right\rangle_{i}}{\left\|a_{j}-a_{i}\right\|}=-2\left\|x_{2}-x_{1}\right\|<0 .
$$

Since $\left\langle x_{2}-x_{1}, a_{j}-a_{i}\right\rangle_{s} \geq\left\langle x_{2}-x_{1}, a_{j}-a_{i}\right\rangle_{i}$, we have

$$
\sum_{\substack{i=1 \\ i \neq j}}^{n}\left(\frac{\left\langle x_{2}-x_{1}, a_{j}-a_{i}\right\rangle_{s}-\left\langle x_{2}-x_{1}, a_{j}-a_{i}\right\rangle_{i}}{\left\|a_{j}-a_{i}\right\|}\right) \geq 0
$$

which contradicts (4) and thus proves the corollary.

Definition 2.11 Let $X$ be a normed linear space and the subsets of distinct points $\mathcal{A}=$ $\left\{a_{1}, \ldots, a_{n}\right\}$ and $\mathcal{B}=\left\{b_{1}, \ldots, b_{n}\right\}$. The subsets $\mathcal{A}$ and $\mathcal{B}$ are isometrically equivalent if there exists a distance preserving a bijective function $F: X \rightarrow X$ which satisfies $F(\mathcal{A})=\mathcal{B}$.

Without loss of generality, we suppose that $F\left(a_{i}\right)=b_{i}$ for all $i \in\{1, \ldots, n\}$. With regards to this definition, we have the following propositions. We omit the proofs.

Proposition 2.12 Let $\left\{a_{1}, \ldots, a_{n}\right\}$ and $\left\{b_{1}, \ldots, b_{n}\right\}$ be two sets of distinct points in $X$ which are isometrically equivalent and let $F$ be the mapping which establishes the equivalence. Then the following statements are equivalent:

(i) $x_{0} \in \mathcal{T}_{X}\left\{a_{1}, \ldots, a_{n}\right\}$;

(ii) $F\left(x_{0}\right) \in \mathcal{T}_{X}\left\{b_{1}, \ldots, b_{n}\right\}$,

where $x_{0} \in X$.

Proposition 2.13 Let $\left\{a_{1}, \ldots, a_{n}\right\}$ be a set of distinct points in the normed linear space $X$.

Then the sets

$$
\left\{a_{1}, \ldots, a_{n}\right\} \text { and }\left\{a_{1}-a_{i}, \ldots, a_{i-1}-a_{i}, 0, a_{i+1}-a_{i}, \ldots, a_{n}-a_{i}\right\}
$$

for $i=\overline{1, n}$ are equivalent. 
The proof follows by observing that the map $F_{i}: X \rightarrow X, F_{i}(x)=x-a_{i}$ is isometric in $X$ for all $i=\overline{1, n}$.

\section{The existence of Torricellian points}

We start with the following known result (cf. Papini and Puerto [9, Proposition 2.3]).

Proposition 3.1 If $X$ is a dual space, in particular, if $X$ is reflexive, then for all $\left\{a_{1}, \ldots, a_{n}\right\}$ a set of distinct points in $X$, the Torricellian set $\mathcal{T}_{X}\left\{a_{1}, \ldots, a_{n}\right\}$ is nonempty in $X$.

Remark 3.2 Veselý [8] proved that for any non-reflexive Banach space, there is an equivalent norm such that for some sets of three points the Torricellian point does not exist.

Furthermore, Papini and Puerto [9, Theorem 2.1] proved the following result.

Proposition 3.3 If $X=c_{0}$, then for every set $\left\{a_{1}, \ldots, a_{n}\right\}$, the Torricellian set $\mathcal{T}_{X}\left\{a_{1}, \ldots, a_{n}\right\}$ is nonempty in $X$.

In what follows, we present a result for the existence for normed linear spaces which are not necessarily reflexive. We start with the following theorem.

Theorem 3.4 Let $(X,\|\cdot\|)$ be a normed linear space and let $\mathcal{A}=\left\{a_{1}, \ldots, a_{n}\right\}$ be a set of distinct points in $X$. If $\mathcal{A} \subset Y$ and $Y \subset X$ is a non-expansive linear subspace in $X$, i.e. there exists a mapping $P: X \rightarrow Y$ such that:

(i) $P$ is the identity of $Y$, i.e. $P x=x$ for all $x \in Y$;

(ii) $\|P x-P y\| \leq\|x-y\|$ for all $x, y \in X$,

then we have the inclusion

$$
\mathcal{T}_{Y}\left\{a_{1}, \ldots, a_{n}\right\} \subseteq \mathcal{T}_{X}\left\{a_{1}, \ldots, a_{n}\right\}
$$

Proof First of all, we observe that $T(P x) \leq T(x)$ for all $x \in X$, as

$$
T(P x)=\sum_{i=1}^{n}\left\|P x-a_{i}\right\|=\sum_{i=1}^{n}\left\|P x-P a_{i}\right\| \leq \sum_{i=1}^{n}\left\|x-a_{i}\right\|=T(x)
$$

for all $x \in X$. By definition, if $x_{0} \in \mathcal{T}_{Y}\left\{a_{1}, \ldots, a_{n}\right\}$, then $T\left(x_{0}\right) \leq T(x)$ for all $x \in Y$. Now, let $x \in X \backslash Y$. Then $P x \in Y$ and thus $T\left(x_{0}\right) \leq T(P x)$. On the other hand, $T(P x) \leq T(x)$ which gives us $T\left(x_{0}\right) \leq T(x)$ for all $x \in X$, i.e. $x_{0} \in \mathcal{T}_{X}\left\{a_{1}, \ldots, a_{n}\right\}$; this completes the proof.

The following corollary contains a sufficient condition for the existence of the Torricellian points.

Corollary 3.5 Let $(X,\|\cdot\|)$ be a normed linear space and let $\mathcal{A}=\left\{a_{1}, \ldots, a_{n}\right\}$ be a system of distinct points in $X$. If the subspace $T_{n}:=\operatorname{span}\left\{a_{1}, \ldots, a_{n}\right\}$ spanned by the set $\mathcal{A}$ is nonexpansive in $X$, then $\mathcal{T}_{X}\left\{a_{1}, \ldots, a_{n}\right\}$ is nonempty.

Proof Since $T_{n}$ is a finite dimensional space, it implies that $T_{n}$ is reflexive. Thus, by Proposition 3.1, $\mathcal{T}_{T_{n}}\left\{a_{1}, \ldots, a_{n}\right\} \neq \emptyset$; and from Theorem 3.4 it follows that $\mathcal{T}_{T_{n}}\left\{a_{1}, \ldots, a_{n}\right\} \subseteq$ $\mathcal{T}_{X}\left\{a_{1}, \ldots, a_{n}\right\}$, which proves the corollary. 
The following theorem contains an example of non-expansive linear subspaces in inner product spaces.

Theorem 3.6 Let $(X,\langle\cdot, \cdot\rangle)$ be an inner product space. If $G$ is a Čebyševian linear subspace in $X$, i.e. every element $x_{0} \in X$ has a unique best approximant in $G$, then $G$ is non-expansive in $X$.

Proof Let $x_{0} \in X$. If $x_{0} \in G$, then $x_{0}=x_{0}+0$ with $0 \in G^{\perp}$. If $x_{0} \notin G$, then there exists an element $x_{1} \in G$ such that $d\left(x_{0}, G\right)=d\left(x_{0}, x_{1}\right)$, i.e. $\inf _{y \in G}\left\|x_{0}-y\right\|=\left\|x_{0}-x_{1}\right\|$. Set $x_{2}:=x_{0}-x_{1}$. Then for all $\lambda \in \mathbb{R}$ and $y \in G$, we have

$$
\left\|x_{2}+\lambda y\right\|=\left\|x_{0}-x_{1}+\lambda y\right\| \geq\left\|x_{0}-x_{1}\right\|=\left\|x_{2}\right\|
$$

for all $\lambda \in \mathbb{R}$ and $y \in G$, which implies that $\left\langle x_{2}, y\right\rangle=0$ for all $y \in G$, i.e. $x_{2} \in G^{\perp}$. Thus, for all $x \in X$, there exist $x_{1} \in G$ and $x_{2} \in G^{\perp}$ such that $x=x_{1}+x_{2}$. We note that this decomposition is unique. If $x=x_{1}+x_{2}, x=y_{1}+y_{2}$ with $x_{1}, y_{1} \in G$ and $x_{2}, y_{2} \in G^{\perp}$, then $G \ni x_{1}-y_{1}=y_{2}-x_{2} \in$ $G^{\perp}$ and since $G \cap G^{\perp}=\{0\}$, we get that $x_{1}=y_{1}$ and $x_{2}=y_{2}$. Define the projection of $X$ on $G$, i.e. the mapping $P: X \rightarrow G$ given by $P(x)=x_{1}$, where $x_{1}$ is the best approximation of $x$ in $G$. Then $P$ is the identity on $G$, and for all $x, y \in X$ we can write

$$
\|x-y\|^{2}=\left\|x_{1}+x_{2}-y_{1}-y_{2}\right\|^{2}=\left\|\left(x_{1}-y_{1}\right)+\left(x_{2}-y_{2}\right)\right\|^{2} .
$$

Since $\left(x_{1}-y_{1}\right) \perp\left(x_{2}-y_{2}\right)$, then by the Pythagorean identity we get

$$
\left\|\left(x_{1}-y_{1}\right)+\left(x_{2}-y_{2}\right)\right\|^{2}=\left\|x_{1}-y_{1}\right\|^{2}+\left\|x_{2}-y_{2}\right\|^{2} \geq\left\|x_{1}-y_{1}\right\|^{2}=\|P x-P y\|^{2}
$$

and thus we obtain the desired inequality $\|P x-P y\| \leq\|x-y\|$, and the theorem is proved.

The above theorem gives us the following result of existence for the Torricellian points (cf. Dragomir and Comǎnescu [7]).

Proposition 3.7 Let $(X,\langle\cdot, \cdot\rangle)$ be an inner product space. Then, for all $\left\{a_{1}, \ldots, a_{n}\right\}$, a set of distinct points in $X, \mathcal{T}_{X}\left\{a_{1}, \ldots, a_{n}\right\}$ is nonempty.

Proof Consider the space $T_{n}:=\operatorname{span}\left\{a_{1}, \ldots, a_{n}\right\}$ which is Čebyševian and non-expansive on $X$ (since $X$ is an inner product space). Then $\mathcal{T}_{T_{n}}\left\{a_{1}, \ldots, a_{n}\right\}$ is nonempty and

$$
\mathcal{T}_{T_{n}}\left\{a_{1}, \ldots, a_{n}\right\} \subseteq \mathcal{T}_{X}\left\{a_{1}, \ldots, a_{n}\right\}
$$

which proves the statement.

\section{The case of collinear points}

In this section, we consider the Torricellian points for a set of collinear points in a normed space. 
Definition 4.1 The set of $n$ distinct points $\left\{a_{1}, \ldots, a_{n}\right\}$ are collinear in the real normed space if there exist two distinct elements $a, b \in X$ and $\lambda_{1}, \ldots, \lambda_{n} \in \mathbb{R}$ such that

$$
a_{i}=\lambda_{i} a+\left(1-\lambda_{i}\right) b, \quad i=\overline{1, n} .
$$

Without loss of generality, we assume that $\lambda_{1}<\cdots<\lambda_{n}$. The following proposition holds.

Proposition 4.2 Let $(X,\|\cdot\|)$ be a real normed space and let $a_{1}, \ldots, a_{2 k+1}$ be $2 k+1$ be collinear points. Then $\mathcal{T}_{X}\left\{a_{1}, \ldots, a_{2 k+1}\right\}=\left\{a_{k+1}\right\}$.

Proof We have successively

$$
\begin{aligned}
T\left(a_{k+1}\right)=\sum_{i=1}^{2 k+1}\left\|a_{k+1}-a_{i}\right\| & =\sum_{i=1}^{2 k+1}\left\|\lambda_{k+1} a+\left(1-\lambda_{k+1}\right) b-\lambda_{i} a-\left(1-\lambda_{i}\right) b\right\| \\
& =\sum_{i=1}^{2 k+1}\left|\lambda_{k+1}-\lambda_{i}\right|\|a-b\| \\
& =\sum_{i=1}^{k}\left(\lambda_{2 k+2-i}-\lambda_{i}\right)\|a-b\|=\sum_{i=1}^{k}\left\|a_{2 k+2-i}-a_{i}\right\| .
\end{aligned}
$$

Now, assume that $x \in X \backslash\left\{a_{k+1}\right\}$. Then, for all $i \in\{1, \ldots, k\}$, one has the inequality

$$
\left\|x-a_{i}\right\|+\left\|x-a_{2 k+2-i}\right\| \geq\left\|a_{2 k+2-i}-a_{i}\right\| .
$$

Since $\left\|x-a_{k+1}\right\|>0$, we get

$$
T(x)=\sum_{i=1}^{k}\left(\left\|x-a_{i}\right\|+\left\|x-a_{2 k+2-i}\right\|\right)+\left\|x-a_{k+1}\right\|>\sum_{i=1}^{k}\left\|a_{2 k+2-i}-a_{i}\right\|=T\left(a_{k+1}\right) .
$$

Consequently, $T(x)>T\left(a_{k+1}\right)$ for all $x \in X \backslash\left\{a_{k+1}\right\}$, which shows that $a_{k+1}$ is the unique Torricellian point associated with $\left\{a_{1}, \ldots, a_{2 k+1}\right\}$.

The following proposition holds.

Proposition 4.3 Let $(X,\|\cdot\|)$ be a normed linear space and let $\left\{a_{1}, \ldots, a_{2 k}\right\}$ be a set of $2 k$ collinear distinct points in $X$. Then the interval $\left[a_{k}, a_{k+1}\right]$ is a subset of $\mathcal{T}_{X}\left\{a_{1}, \ldots, a_{2 k}\right\}$.

Proof Let $x \in\left[a_{k}, a_{k+1}\right]$. Then $x=\lambda a+(1-\lambda) b$ with $\lambda \in\left[\lambda_{k}, \lambda_{k+1}\right]$. A similar computation to that of the proof of Proposition 4.2 shows that

$$
T(x)=\sum_{i=1}^{k}\left\|a_{2 k+1-i}-a_{i}\right\|
$$

Now, suppose that $y \in X$. Then

$$
T(y)=\sum_{i=1}^{2 k}\left\|y-a_{i}\right\|=\sum_{i=1}^{k}\left(\left\|y-a_{i}\right\|+\left\|y-a_{2 k+1-i}\right\|\right) \geq \sum_{i=1}^{k}\left\|a_{i}-a_{2 k+1-i}\right\|=T(x),
$$

which shows that $\left[a_{k}, a_{k+1}\right] \subset \mathcal{T}_{X}\left\{a_{1}, \ldots, a_{2 k}\right\}$. 
We use the following notation in the following characterisation theorem. Let $a$ and $b$ be two distinct vectors in $X$, we define

$$
d r(a, b):=\{\lambda a+(1-\lambda) b \mid \lambda \in \mathbb{R}\}
$$

Theorem 4.4 Let $(X,\|\cdot\|)$ be a real normed space. The following statements are equivalent:

(i) $(X,\|\cdot\|)$ is strictly convex.

(ii) For every $k \in \mathbb{N}$ and for every $\left\{a_{i}, \ldots, a_{2 k}\right\}, 2 k$ collinearly distinct point in $X$, we have

$$
\mathcal{T}_{X}\left\{a_{1}, \ldots, a_{2 k}\right\}=\left[a_{k}, a_{k+1}\right]
$$

(iii) For every distinct points $a_{1}$ and $a_{2}$ in $X$, we have $\mathcal{T}_{x}\left\{a_{1}, a_{2}\right\}=\left[a_{1}, a_{2}\right]$.

Proof '(i) $\Longrightarrow$ (ii)'.

Let $(X,\|\cdot\|)$ be a strictly convex normed space and let $\left\{a_{1}, \ldots, a_{2 k}\right\}$ be a set of $2 k$ collinearly distinct points in $X$. By Proposition 4.3 we have that $\left[a_{k}, a_{k+1}\right] \subset \mathcal{T}_{X}\left\{a_{1}, \ldots, a_{2 k}\right\}$, so we want to show that $\mathcal{T}_{X}\left\{a_{1}, \ldots, a_{2 k}\right\} \subset\left[a_{k}, a_{k+1}\right]$. Let $x \in \mathcal{T}_{X}\left\{a_{1}, \ldots, a_{2 k}\right\}$ and suppose that $\left\|x-a_{k}\right\|+\left\|x-a_{k+1}\right\|>\left\|a_{k}-a_{k+1}\right\|$. We have

$$
\begin{aligned}
T(x)=\sum_{i=1}^{2 k}\left\|x-a_{i}\right\| & =\sum_{i=1}^{k-1}\left(\left\|x-a_{i}\right\|+\left\|x-a_{i+k+1}\right\|\right)+\left\|x-a_{k}\right\|+\left\|x-a_{k+1}\right\| \\
& >\sum_{i=1}^{k-1}\left(\left\|x-a_{i}\right\|+\left\|x-a_{i+k+1}\right\|\right)+\left\|a_{k}-a_{k+1}\right\|=T\left(x_{0}\right),
\end{aligned}
$$

where $x_{0} \in\left[a_{k}, a_{k+1}\right]$ (see Proposition 4.2), which contradicts the fact that $x$ minimises the Torricellian map $T$. Hence $\left\|x-a_{k}\right\|+\left\|x-a_{k+1}\right\|=\left\|a_{k}-a_{k+1}\right\|$, and $x-a_{k}=\theta\left(a_{k+1}-x\right)$ for some nonnegative $\theta$. It follows that $x=\frac{1}{1+\theta} a_{k}+\frac{\theta}{1+\theta} a_{k+1} \in\left[a_{k}, a_{k+1}\right]$.

'(ii) $\Longrightarrow$ (iii)'. The proof of this implication is trivial, so we omit the details.

'(iii) $\Longrightarrow$ (i)'. Suppose that $(X,\|\cdot\|)$ is not strictly convex, i.e. there exist two elements $x, y \in X, x \neq y$, such that $\|x+y\|=\|x\|+\|y\|$ and $x, y$ are linearly independent. Because of the linear independence, $x \neq-y$ and if we apply statement (ii) for $a_{1}=x$ and $a_{2}=-y$, we can write

$$
\mathcal{T}_{X}\{x,-y\}=[x,-y]
$$

Now, $0 \notin[x,-y]$ as if we assume that $0 \in[x,-y]$, then there exists $\lambda_{0} \in[0,1]$ such that

$$
\lambda_{0} x+\left(1-\lambda_{0}\right)(-y)=0,
$$

i.e. the system of vectors $\{x, y\}$ is linearly dependent, which contradicts the above assumption. Since $\mathcal{T}_{X}\{x,-y\}=[x,-y]$ and $0 \notin[x,-y]$, then we can state 
where $x_{0} \in[x,-y]$. However,

$$
T(0)=\|x\|+\|y\|
$$

and

$$
\begin{aligned}
T\left(x_{0}\right) & =\left\|x-\left(\lambda_{0} x+\left(1-\lambda_{0}\right)(-y)\right)\right\|+\left\|-y-\left(\lambda_{0} x+\left(1-\lambda_{0}\right)(-y)\right)\right\| \\
& =\left(1-\lambda_{0}\right)\|x+y\|+\lambda_{0}\|x+y\|=\|x+y\|,
\end{aligned}
$$

where $\lambda_{0} \in[0,1]$. Let us observe that by the inequality (6) we deduce $\|x\|+\|y\|>\|x+y\|$, which contradicts the hypothesis $\|x\|+\|y\|=\|x+y\|$, hence $(X,\|\cdot\|)$ is strictly convex.

Corollary 4.5 Let $(X,\langle\cdot, \cdot\rangle)$ be an inner product space and let $\left\{a_{1}, \ldots, a_{2 k}\right\}$ be $2 k$ collinearly distinct points in $X$. Then $\mathcal{T}_{X}\left\{a_{1}, \ldots, a_{2 k}\right\}=\left[a_{k}, a_{k+1}\right]$.

\section{The uniqueness of Torricellian points for non-collinear sets}

In this section, we recall and present results concerning uniqueness of the set of Torricellian points.

Proposition 5.1 Let $(X,\|\cdot\|)$ be a normed linear space and let $\mathcal{A}=\left\{a_{1}, \ldots, a_{n}\right\} \subset X(n \geq 3)$ be a set of non-collinear points in $X$. If the space is strictly convex, then $\mathcal{T}_{X}\left\{a_{1}, \ldots, a_{n}\right\}$ contains at most one element.

Proof Suppose that there exist two distinct elements $x_{1}, x_{2} \in \mathcal{T}_{X}\left\{a_{1}, \ldots, a_{n}\right\}$. Then $T\left(x_{1}\right)=$ $T\left(x_{2}\right)=\inf _{x \in X} T(x)$. Let $\lambda \in(0,1)$ and put $x_{\lambda}:=\lambda x_{1}+(1-\lambda) x_{2}$. As $T$ is a strictly convex mapping on $X$ (see Proposition 2.5), we have that

$$
T\left(x_{\lambda}\right)<\lambda T\left(x_{1}\right)+(1-\lambda) T\left(x_{2}\right)=\inf _{x \in X} T(x),
$$

which produces a contradiction and the theorem is thus proved.

We remark that Proposition 5.1 is also proved by Papini and Puerto [9] in their Remark 3.1.

The following corollary recaptures the result in Dragomir and Comănescu [7, Theorem 1].

Corollary 5.2 Let $(X ;(\cdot, \cdot))$ be an inner product space and let $\mathcal{A}=\left\{a_{1}, \ldots, a_{n}\right\} \subset X(n \geq 3)$ be a set of non-collinear points in $X$. Then $\mathcal{T}_{X}\left\{a_{1}, \ldots, a_{n}\right\}$ contains a unique point.

Proof The existence follows by Theorem 3.7, while the uniqueness follows by Theorem 5.1, taking into account that every inner product space is a strictly convex space.

Martini et al. [5] showed that the uniqueness of the Torricellian point characterises strict convexity in Minkowski spaces. In the next proposition, we show that there exists a noncollinear set, for which the Torricellian point is unique, but the normed space is not strictly convex, thus showing that the uniqueness property does not characterise strict convexity. 
We note that the definition of collinear in [5] is metric dependent. Thus, Proposition 5.1 only generalises their result when the metric agrees with our definition of collinearity ( $c f$. Definition 4.1).

Proposition 5.3 Let $(X,\|\cdot\|)$ be a normed space which is not strictly convex. There exists a set $\mathcal{A}=\left\{a_{1}, a_{2}, a_{3}\right\}$ of non-collinear distinct points in $X$ such that $\mathcal{T}_{X}\left\{a_{1}, a_{2}, a_{3}\right\}$ has at most one element.

Proof Since $X$ is not strictly convex, by the proof of Theorem 4.4 ('(iii) $\Rightarrow$ (i)'), there exist $a_{1}, a_{2}$ distinct points such that $\mathcal{T}_{X}\left\{a_{1}, a_{2}\right\} \backslash\left[a_{1}, a_{2}\right] \neq \emptyset$. Let $a_{3} \in \mathcal{T}_{X}\left\{a_{1}, a_{2}\right\} \backslash\left[a_{1}, a_{2}\right]$. We observe that $a_{3} \notin d r\left(a_{1}, a_{2}\right)$, which implies that $a_{1}, a_{2}, a_{3}$ are non-collinear distinct points. We have $T_{\mathcal{A}}\left(a_{3}\right)=\left\|a_{3}-a_{1}\right\|+\left\|a_{3}-a_{2}\right\|=\left\|a_{1}-a_{2}\right\|$. If $x \neq a_{3}$, then $\left\|x-a_{3}\right\|>0$ and $\left\|x-a_{1}\right\|+$ $\left\|x-a_{2}\right\| \geq\left\|a_{1}-a_{2}\right\|$ and consequently, $T_{\mathcal{A}}(x)>T_{\mathcal{A}}\left(a_{3}\right)$. Hence, $\mathcal{T}_{X}\left\{a_{1}, a_{2}, a_{3}\right\}=\left\{a_{3}\right\}$.

\section{Open problems}

Suppose we define the property $\mathcal{P}_{3}$ to be the following: For all the sets $\left\{a_{1}, a_{2}, a_{3}\right\}$ of distinct points, the set $\mathcal{T}_{X}\left\{a_{1}, a_{2}, a_{3}\right\}$ has at most one element. We then ask the following question: If the property $\mathcal{P}_{3}$ holds true in a normed space $X$, can we affirm that $X$ is strictly convex? The answer is no, and is verified by Corollary 6.4 of Section 6. Furthermore, we ask the question: If we generalise the property $\mathcal{P}_{3}$ to $\mathcal{P}_{n}$, where $n \geq 3$, for what values of $n$ can we affirm that $X$ is strictly convex? In the next example, we show that when $\mathcal{P}_{n}$ holds true in a normed space $X$ and $n$ is an odd number, $X$ is not necessarily strictly convex.

Example 5.4 If we consider the space $\left(\mathbb{R}^{d},\|\cdot\|_{1}\right)$ (which is not strictly convex), then for all sets $\left\{a_{1}, \ldots, a_{n}\right\}$ (where $n$ is an odd number) of distinct points, $\mathcal{T}_{X}\left\{a_{1}, \ldots, a_{n}\right\}$ has at most one element (cf. Section 6).

It remains as an open problem at this point for the case of $n \geq 3$, where $n$ is an even number.

\section{The Torricellian points in the Taxicab geometry}

In this section we study the Torricellian points of the set $\mathcal{A}$ in the Taxicab geometry. We consider the real vector space $\mathbb{R}^{d}$. The Taxicab norm (or Manhattan norm) $\|\cdot\|_{1}$ gives rise to the Taxicab metric. The Taxicab norm is defined by

$$
\left\|\left(x_{1}, \ldots, x_{d}\right)\right\|_{1}=\sum_{i=1}^{d}\left|x_{i}\right|
$$

Let be $\mathbf{a}_{1}=\left(a_{1}^{1}, \ldots, a_{1}^{d}\right), \ldots, \mathbf{a}_{n}=\left(a_{n}^{1}, \ldots, a_{n}^{d}\right)$. We note by $\mathcal{A}=\left\{\mathbf{a}_{1}, \ldots, \mathbf{a}_{n}\right\} \subset \mathbb{R}^{d}$. We introduce the following notation:

$$
\mathbf{A}^{i}=\left(a_{1}^{i}, \ldots, a_{n}^{i}\right) \in \mathbb{R}^{n} \quad \text { for all } i \in\{1, \ldots, d\}
$$

If $\mathbf{B}=\left(b^{1}, \ldots, b^{n}\right) \in \mathbb{R}^{n}$, then

$$
\operatorname{midd}(\mathbf{B})= \begin{cases}\left\{b^{\sigma(k+1)}\right\}, & n=2 k+1, \\ {\left[b^{\sigma(k)}, b^{\sigma(k+1)}\right],} & n=2 k\end{cases}
$$


where $\sigma:\{1, \ldots, n\} \rightarrow\{1, \ldots, n\}$ is a permutation which satisfies the property $\mathcal{P}: b^{\sigma(1)} \leq$ $b^{\sigma(2)} \leq \cdots \leq b^{\sigma(n)}$. We note that the set midd(B) does not depend on the permutation $\sigma$ with the property $\mathcal{P}$.

\subsection{The case $d=1$}

We consider the more general Torricelli problem, i.e. to minimise the functional $T_{\mathbf{A}}: \mathbb{R} \rightarrow$ $\mathbb{R}$,

$$
T_{\mathbf{A}}(x)=\sum_{i=1}^{n}\left|x-a^{i}\right|
$$

where $\mathbf{A}=\left(a^{1}, \ldots, a^{n}\right) \in \mathbb{R}^{n}$ in which the points $a^{1}, \ldots, a^{n}$ are not necessary distinct. Using similar arguments to those in Proposition 4.2, Proposition 4.3 and Theorem 4.4, we obtain the following result.

Theorem 6.1 The set of Torricellian points of $\mathbf{A} i s$

$$
\mathcal{T}_{\mathbb{R}}(\mathbf{A})=\operatorname{midd}(\mathbf{A})
$$

\section{Remark 6.2}

(i) We have a unique Torricellian point if and only if midd(A) has a unique point;

(ii) If $n$ is odd, then the set of Torricellian points has a unique element.

\subsection{The general case $d>1$}

The Torricellian functional has the form

$$
T\left(x_{1}, \ldots, x_{d}\right)=\sum_{i=1}^{d} \sum_{j=1}^{n}\left|x_{i}-a_{i}^{j}\right|=\sum_{i=1}^{d} T_{\mathbf{A}^{i}}\left(x_{i}\right) .
$$

By Theorem 6.1 we have the following.

Theorem 6.3 The set of Torricellian points of $\mathcal{A}$ is

$$
\mathcal{T}_{\mathbb{R}^{d}}\left\{\mathbf{a}_{1}, \ldots, \mathbf{a}_{n}\right\}=\operatorname{midd}\left(\mathbf{A}^{1}\right) \times \operatorname{midd}\left(\mathbf{A}^{2}\right) \times \cdots \times \operatorname{midd}\left(\mathbf{A}^{d}\right)
$$

\section{Corollary 6.4}

(i) If $n$ is odd, then $\mathcal{T}_{\mathbb{R}^{d}}\left\{\mathbf{a}_{1}, \ldots, \mathbf{a}_{n}\right\}$ has a unique point;

(ii) If $n$ is even, then $\mathcal{T}_{\mathbb{R}^{d}}\left\{\mathbf{a}_{1}, \ldots, \mathbf{a}_{n}\right\}$ has a unique point if and only if all the sets $\operatorname{midd}\left(\mathbf{A}^{i}\right)$ have a unique element.

We present two sets of Torricellian points in the space $\left(\mathbb{R}^{2},\|\cdot\|_{1}\right)$ : the first has one element and the second has an infinite number of elements.

Example 6.5 The set of Torricellian points of $\mathcal{A}_{I}=\{(1,0),(-1,0),(0,1),(0,-1)\} \subset \mathbb{R}^{2}$ is

$$
\mathcal{T}_{\mathcal{A}_{I}}=\{(0,0)\}
$$


Example 6.6 The set of Torricellian points of $\mathcal{A}_{I I}=\{(1,1),(-1,1),(-1,-1),(1,-1)\} \subset \mathbb{R}^{2}$ is

$$
\mathcal{T}_{\mathcal{A}_{I I}}=[-1,1] \times[-1,1]
$$

\section{Conclusions}

We consider the Torricelli problem in the settings of normed spaces. We recall some known results such as the existence of Torricellian point(s) in reflexive spaces. Our result shows that when the set $\mathcal{A}$ spans a non-expansive linear subspace of a normed space, the Torricellian point(s) exists. This implies that the Torricellian point of a set $\mathcal{A}$ always exists in inner product spaces.

Furthermore, we show that in any normed space, if all points in the set $\mathcal{A}=\left\{a_{1}, \ldots, a_{2 k+1}\right\}$ lie in a single straight line (collinear), then the Torricellian set is the singleton $\left\{a_{k+1}\right\}$. When all the points in the set $\mathcal{A}=\left\{a_{1}, \ldots, a_{2 k}\right\}$ are collinear, the Torricellian set contains the segment $\left[a_{k}, a_{k+1}\right]$. This result can be used to characterise strictly convex spaces. When the Torricellian set of the collinear set $\left\{a_{1}, \ldots, a_{2 k}\right\}$ is equal to the segment $\left[a_{k}, a_{k+1}\right]$, then the space must be a strictly convex space and vice versa. This implies that when the space is an inner product space, the Torricellian set of the collinear set $\left\{a_{1}, \ldots, a_{2 k}\right\}$ is the segment $\left[a_{k}, a_{k+1}\right]$.

We discuss the uniqueness of the Torricellian points for non-collinear sets. We show that the Torricellian point is unique when the space is strictly convex. However, we show the uniqueness does not characterise strict convexity. Lastly, we consider some examples of the Torricellian points in a normed space endowed with the Taxicab geometry.

Competing interests

The authors declare that they have no competing interests.

\section{Authors' contributions}

SSD, DC and EK contributed equally in all stages of writing the paper. All authors read and approved the final manuscript.

\section{Author details}

${ }^{1}$ School of Engineering and Science, Victoria University, P.O. Box 14428, Melbourne, Victoria 8001, Australia. ${ }^{2}$ School of Computational and Applied Mathematics, University of the Witwatersrand, Private Bag 3, Wits, Johannesburg, 2050, South Africa. ${ }^{3}$ University of the West Timisoara, B-dul V. Parvan 4, Timisoara, 300233, Romania.

\section{Acknowledgements}

Dan Comǎnescu has been supported by the grant of the Romanian National Authority for Scientific Research, CNCS UEFISCDI, project number PN-II- RU-TE-2011-3-0006

Received: 25 February 2013 Accepted: 6 May 2013 Published: 22 May 2013

\section{References}

1. Dalla, L: A note on the Fermat-Torricellian point of a d-simplex. J. Geom. 70, 38-43 (2001)

2. Simons, S: Some features of the general Fermat point. Math. Gaz. 87(508), 60-70 (2003)

3. Kupitz, YS, Martini, H: Geometric aspects of the generalized Fermat-Torricelli problem. In: Intuitive Geometry (Budapest, 1995). Bolyai Soc. Math. Stud., vol. 6, pp. 55-127 (1997)

4. Boltyanski, V, Martini, H, Soltan, V: Geometric Methods and Optimization Problems. Combinatorial Optimization, vol. 4. Kluwer Academic, Dordrecht (1999)

5. Martini, H, Swanepoel, KJ, Weiss, G: The Fermat-Torricelli problem in normed planes and spaces. J. Optim. Theory Appl. 115(2), 283-314 (2002)

6. Dragomir, SS, Comănescu, D, Cho, YJ, Kim, SS: On Torricelli's problems in inner product space. Aust. Math. Soc. Gaz. 27(4), 173-180 (2000)

7. Dragomir, SS, Comănescu, D: On the Torricellian point in inner product spaces. Demonstr. Math. 41(3), 639-650 (2008)

8. Veselý, L: A characterization of reflexivity in the terms of the existence of generalized centers. Extr. Math. 8(2-3), 125-131 (1993)

9. Papini, PL, Puerto, J: Averaging the k largest distances among $n$ : k-centra in Banach spaces. J. Math. Anal. Appl. 291(2), 477-487 (2004)

10. Dragomir, SS: Semi-inner Products and Applications. Nova Science Publishers, New York (2004) 
doi:10.1186/1029-242X-2013-258

Cite this article as: Dragomir et al.: Torricellian points in normed linear spaces. Journal of Inequalities and Applications 2013 2013:258.

Submit your manuscript to a SpringerOpen ${ }^{\circ}$ journal and benefit from:

- Convenient online submission

- Rigorous peer review

- Immediate publication on acceptance

Open access: articles freely available online

- High visibility within the field

- Retaining the copyright to your article

Submit your next manuscript at $\gg$ springeropen.com 